EPJ Web of Conferences 28, 02002 (2012)

DOI: $10.1051 /$ epjconf/20122802002

(C) Owned by the authors, published by EDP Sciences, 2012

\title{
Results from TOTEM and ALFA
}

Karsten Eggert ${ }^{1, a}$

Case Western Reserve University, Department of Physics - Cleveland, OH, USA

Abstract. Results from TOTEM and ALFA

\section{The ALFA detector}

[1] [2] [3] [4]

\section{The TOTEM detector}

[5] [6]

\section{The TOTEM physics results}

[7] [8]

\section{References}

1. F. Anghinolfi et al., JINST 2 (2007) P07004

2. B. Di Girolamo, Nucl. Instrum. Meth. A581 (2007) 526 $-530$

3. P. Jenni et al., ATLAS Forward Detectors for Measurement of Elastic Scattering and Luminosity (CERN, Geneva 2008) CERN-LHCC-2008-004

4. S. Franz and P. Barillon, Nucl. Instrum. Meth. A610 (2009) $35-40$

5. V. Berardi et al. ((TOTEM Collaboration), Total crosssection, elastic scattering and diffraction dissociation at the Large Hadron Collider at CERN : TOTEM Technical Design Report (CERN, Geneva 2004) CERN-LHCC2004-002

6. G. Anelli et al. (TOTEM Collaboration), JINST 3 (2008) S08007

7. G. Antchev et al. (TOTEM Collaboration), Europhys. Lett. 95 (2011) 41001

8. G. Antchev et al. (TOTEM Collaboration), Europhys. Lett. 96 (2011) 21002

\footnotetext{
a e-mail: Karsten.Eggert@cern.ch
} 